\title{
The Public Health Community Platform: Shared Resources For Enterprise Solutions
}

\author{
Marcus Rennick*1, Scott Gordon ${ }^{1}$, Monica Huang ${ }^{1}$, Anita Samuel ${ }^{1}$, Paula Soper ${ }^{1}$ and Laura \\ Conn ${ }^{2}$
}

${ }^{1}$ Informatics, Association of State and Territorial Health Officials, Arlington, VA, USA; ${ }^{2}$ Centers for Disease Control and Prevention, Atlanta, GA, USA

\section{Objective}

To update the public health practice community on the continuing development of the Public Health Community Platform (PHCP).

\section{Introduction}

Public health is at a precipice of increasing demand for the consumption and analysis of large amounts of disparate data, the centralization of local and state IT offices, and the compartmentalization of programmatic technology solutions. Public health informatics needs differ across programmatic areas, but may have commonalities across jurisdictions. Initial development of the PHCP was launched with the goal of providing a shared infrastructure for state and local jurisdictions enabling the development of interoperable systems and distributed analytical methods with common sources of data. The PHCP is being designed to leverage recent successes with cloud-based technology in public health.

Success of the PHCP is dependent on the involvement of state and local public health jurisdictions in the transparent development and future direction of the platform. Equally critical to success is the selection of appropriate technology, consideration of various governance structures, and full understanding of the legal implications of a shared platform model.

\section{Methods}

The Association of State and Territorial Health Officials (ASTHO), under a cooperative agreement with the Centers for Disease Control and Prevention (CDC), is coordinating an ongoing national effort to create the framework of the PHCP. A Steering Committee (an interim advisory board) was initially formed to provide an overarching advisory role in designing various assessments and identifying use cases for the proposed PHCP. The Steering Committee met regularly, both in-person and virtually, throughout the initial phase of development to refine the direction of the PHCP and help guide the work of the assessment teams (technical, governance, communication, and use cases).

\section{Results}

Technical Assessment:

The MIT- Lincoln Laboratory (MIT-LL) provided a roadmap for development, an assessment of existing technology, and an expansive analysis of risk and mitigation for various modes of data sharing. The suggested roadmap focused on the premise that users adopt a system that serves an immediate need and improves workflow efficiency. This immediate need necessitates seeding any platform development with explicit functionality to benefit the individual user. Sharing is secondary.

\section{Governance and Sustainability:}

ASTHO contracted with Clinovations to identify several options for ownership, governance, and sustainability. Each ownership option (federally owned, incubated by a non-profit, or separately incorporated) can institute a form of governance with varying levels of control and community driven consensus. Advantages and disadvantages of several funding models were also enumerated by the contractor.

Communication:

Porter Novelli, a communications firm, was contracted to provide branding and marketing materials for the PHCP. Generally, Porter Novelli worked with the Steering Committee to formulate how to express the concept of the PHCP.

Use Cases:

Two use cases were identified by the Steering Committee: an ELR reportable disease decision trigger (developed by APHL) and a clinical decision support for immunization forecasting (conducted by PHII). PHII also provided a gap analysis between available tools for community health assessments and current needs.

\section{Conclusions}

The assessment teams provided the necessary framework to build a successful community-owned platform for innovative and collaborative public health practice. The PHCP has the potential to provide a single source for public health decision support solutions, reducing the number of redundant systems. Additionally, the platform could be instrumental in providing the implementation space for national pilot informatics projects.

The next year of development will involve implementing Steering Committee decisions, prioritizing use cases that can be advanced as pilots, and building the technical architecture and community to support those pilots.

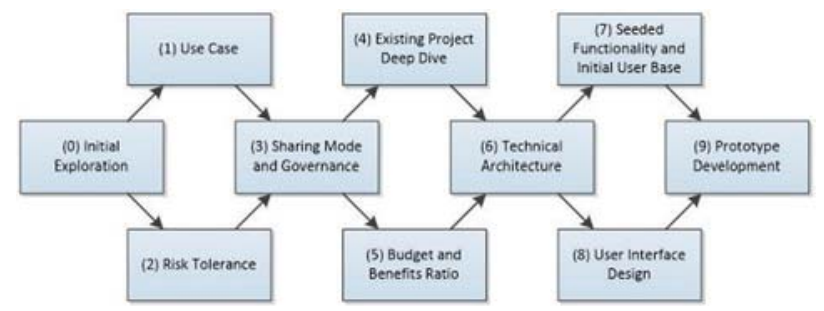

Figure 1. PHCP Development Roadmap

\section{Keywords}

Platform; Informatics; Enterprise; Solutions; interoperability

\section{Acknowledgments}

We would like to acknowledge the tremendous amount of effort from our steering committee members.

\author{
*Marcus Rennick \\ E-mail: mrennick@astho.org
}

